\title{
Loneliness and Social Isolation During the COVID-19 Pandemic
}

\section{A Systematic Review Enriched With Empirical Evidence From a Large-Scale Diary Study}

\author{
Susanne Buecker ${ }^{1}$ ib and Kai T. Horstmann² \\ ${ }^{1}$ Department of Psychology, Ruhr University Bochum, Germany \\ ${ }^{2}$ Department of Psychology, Humboldt-Universität zu Berlin, Germany
}

\begin{abstract}
The outbreak of the global COVID-19 pandemic has drastically altered people's lives. Loneliness and social isolation were publicly discussed as possible psychological consequences of the measures taken to slow the virus spread. These public discussions have sparked a surge in empirical studies on loneliness and social isolation. In this study, we first provide a systematic review synthesizing recent literature on the prevalence and correlates of loneliness and social isolation during the early phase of the COVID-19 pandemic ( $k=53$ studies). We found that most quantitative studies included in the systematic review were cross-sectional. The few longitudinal studies mainly reported increases in loneliness, especially when the pre-pandemic measurement occasions were months or years before the COVID-19 pandemic. Studies with pre-pandemic measures weeks or days before the pandemic reported relatively stable or even decreasing loneliness trends. Second, we enrich the systematic review with an empirical investigation on daily changes in the perceived quality and quantity of social relationships during the pandemic compared to before the pandemic $(N=4,823)$. This empirical investigation showed that, on average, the quality of social relationships was perceived worse during the pandemic than before. This perception got slightly stronger over the first 2 weeks of the pandemic but stagnated thereafter. Regarding the quantity of social relationships, participants reported on average that they had fewer social interactions at the beginning of the study than before the pandemic. This perceived reduction in the quantity of social interactions linearly decreased over time.
\end{abstract}

Keywords: loneliness, COVID-19, relationship quality, relationship quantity, systematic review

On March 11, 2020, the World Health Organization (WHO) declared the outbreak of the global COVID-19 pandemic (WHO, 2020). Most countries have implemented measures against the spread of the virus, such as school closings, contract restrictions, or travel bans. These measures drastically altered people's lives, impacting various aspects of the private, public, and global economy (Xiong et al., 2020) and resulting in uncertainties and worries. These uncertainties and worries, combined with the demand for reduction of physical, social contacts, have been discussed as possible triggers of loneliness (Klein, 2020). Consequently, the topic of (rising) loneliness has attracted public attention, and scientific articles on loneliness during the COVID-19 pandemic have been published numerously.

Perceived social isolation - more colloquially known as loneliness - is commonly defined as an aversive feeling arising when the quality or quantity of one's social relationships is perceived as dissatisfying (Perlman \& Peplau, 1981).
A construct related to but distinct from loneliness is objective social isolation (Holt-Lunstad et al., 2015). Objective social isolation refers to the actual contact frequency or the actual total number of social contacts. Contact restrictions and stay-at-home orders have been speculated to impact the perceived quality and quantity of social relationships, potentially resulting in loneliness. For example, the quality of social relationships may have been affected because many conversations revolved exclusively around COVID-19. Additionally, the number of personal social contacts was reduced to prevent infection with the virus. Alternative forms of communication, such as telephone and video chat instead of face-to-face communication, might have been perceived as a more superficial social interaction resulting in lower perceived closeness and intimacy and higher loneliness.

As the COVID-19 pandemic-related measures might have affected both the quality and the quantity of social 
relationships, an increase in loneliness and social isolation during the pandemic would be plausible. However, the empirical evidence on this assumption is inconclusive. Some studies have found an increase in loneliness, whereas other studies report relatively stable loneliness trends (e.g., Buecker et al., 2020; Luchetti et al., 2020; Stefana et al., 2020). Moreover, it is unclear whether the pandemicrelated measures were associated with changes in the perceived quality of one's social relationships, the quantity of one's social relationships, or both. This part is crucial to understand why pandemic-related contact restrictions might be related to changes in loneliness and social isolation.

The first research objective was to summarize the empirical evidence on the prevalence and changes of loneliness in the context of the COVID-19 pandemic. Furthermore, we examine loneliness correlates during the COVID-19 pandemic and compare them to pre-pandemic findings on loneliness. This systematic review is intended to inform researchers and policymakers whether there has been a general increase in loneliness since the COVID-19 pandemic and whether there are possible at-risk groups that were particularly vulnerable to such an increase.

The second research objective was to enrich the systematic review findings with empirical evidence on changes in the perceived quality and quantity of social relationships from a large-scale daily diary study. Using an intensive longitudinal design, we were able to explore processes of changes in social relationships that improve the understanding of loneliness and social isolation during the COVID-19 pandemic.

\section{Research Objective 1: Systematic Review}

\section{Methods}

We followed the Preferred Reporting Items for Systematic Reviews and Meta-Analyses (PRISMA) guidelines (Moher et al., 2009).

\section{Search Strategy}

We conducted a systematic literature search following the PRISMA 2009 flow diagram (Figure 1) on PsycINFO in October (Week 4) 2020 via the Ovid platform (https:// ovidsp.dc2.ovid.com). After screening search results for eligibility, we updated our search in November (Week 3). We applied the following search terms in the title and abstract: ((COVID-19 OR coronavirus OR sars-cov-2 OR distancing policies) AND (loneliness $O R$ isolation $O R$ alone OR lonely)). Additionally, we performed a manual search on Google Scholar.

\section{Study Selection and Eligibility Criteria}

In Step 1, the first author and a trained undergraduate student screened titles and abstracts of each search result for relevance. In Step 2, full-text articles were accessed for eligibility by two trained undergraduate students based on detailed coding instructions and supervision. Both coders double-coded $50 \%$ of the results. The intercoder agreement was $92 \%$ for exclusion or inclusion of studies, calculated as $100 \times$ (number of consistently included studies/number of all double coded studies). We excluded studies if they: (1) did not measure loneliness or social isolation, (2) used a small sample size $(N<10)$, (3) were not written in German or English language, or (4) were non-empirical articles (e.g., reviews, commentaries). Regarding the coded study characteristics, intercoder agreement ranged from $69 \%$ to $94 \%$.

\section{Data Extraction}

We included the following relevant data: (1) Authors, (2) year of publication, (3) country of origin of the sample, (4) study design, (5) sample type, (6) sample size, (7), mean age, (8) age range, (9) scale used to measure loneliness and social isolation, (10) prevalence of loneliness and social isolation (before and during the pandemic), and (11) correlates of loneliness and social isolation.

\section{Primary Study Quality Assessment}

We used an adapted version of the Newcastle-Ottawa Scale (NOS; Wells et al., 2004) to code each study's primary study quality based on the full-text articles. This scale has been used in a previous systematic review on the impact of the COVID-19 pandemic on mental health outcomes (Xiong et al., 2020). It consists of three dimensions: selection, comparability, and outcome. These dimensions assess the representativeness of the sample, the sample size justification, ascertainments of COVID-19 exposure, comparability of measures before and during the COVID-19 pandemic based on study design or analysis, the quality of the loneliness measure used, and appropriateness of the statistical analysis. We provide further details in the online supplement.

\section{Results}

\section{Search Results}

We identified 300 articles in our standardized literature search. We removed 167 in Step 1, leaving 133 full-text articles for eligibility in Step 2. In this step, 56 articles were excluded for not measuring loneliness. One article was excluded because it had no relation to COVID-19. Two articles were excluded for using a small samples $(N<10)$, and 21 because they were non-empirical. Consequently, 53 articles met the inclusion criteria ( $k=49$ quantitative studies, $k=4$ qualitative studies). References of all included articles 


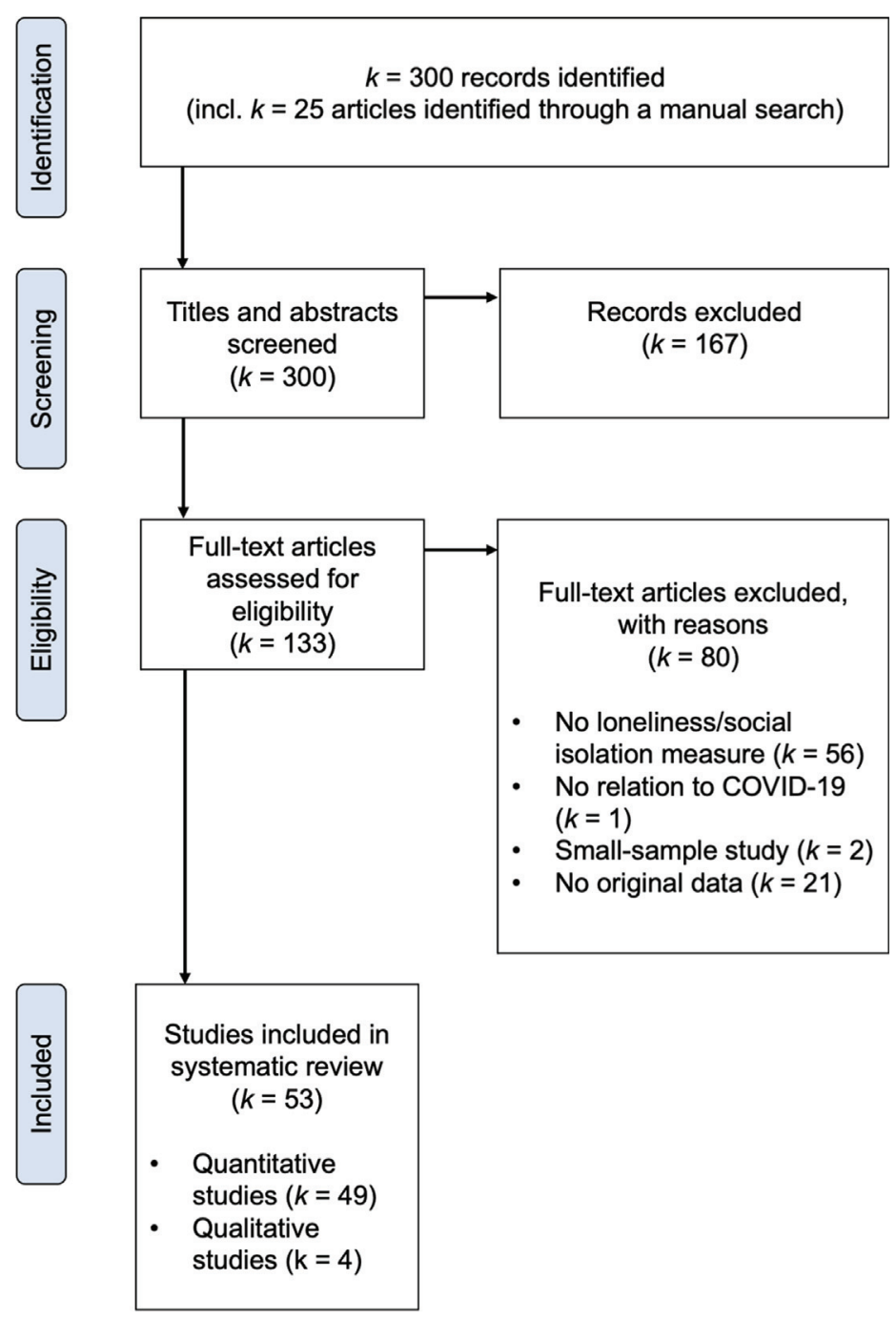

Figure 1. PRISMA flow diagram.

are presented in the online supplementary material (Table S1).

\section{Study Characteristics}

Of all included quantitative studies $(k=49), 13$ studies reported a comparison of the loneliness prevalence before and during the COVID-19 pandemic, of which six also examined correlates of loneliness during the pandemic. In 12 studies, the level of loneliness during the pandemic, but no comparison with the loneliness level before the pandemic was reported. Of those, five studies also examined correlates of loneliness during the pandemic. Twenty-four studies did not report any prevalence of loneliness but examined correlates of loneliness during the pandemic. In addition to these studies, we included $k=4$ qualitative studies.

We summarize the study characteristics and main study findings of all included articles in Table S1 (OSM 1) at the
Open Science Framework (OSF; https://osf.io/w25nk/). The sample sizes ranged from $N=19$ to $N=60,341$ participants. Thirty-six studies were cross-sectional, 13 studies were longitudinal (with at least 2 measurement occasions), and 4 were qualitative studies. The included studies were conducted across 19 different countries. Fourteen studies were conducted in the United States, and 24 studies were conducted across different European countries. Of all included studies that did report information regarding the age of their participants, $k=9$ used an older adult's sample (60+ years), $k=4$ used an age-diverse nationally representative sample, $k=29$ used an age-diverse community sample (convenient), $k=3$ used a student sample (convenient), and $k=1$ used a children sample (below 18 years). Most studies did not report any information regarding the ethnicity of their sample. Of those that did report ethnicity information, $k=9$ studies used predominantly White/European 
samples, and $k=8$ used heterogeneous samples, in which no ethnicity was represented by $70 \%$ or more.

\section{Primary Study Quality Assessment}

The results of the primary study quality assessment are presented in Table S1 (OSM 1). The number of quality stars awarded ranged from 2 to $10(M d n=5)$. Twelve studies reported a representative sample. A satisfactory and/or justified sample size was used in 45 studies. In 35 studies, loneliness was assessed with a validated loneliness scale. The majority of quantitative studies clearly described the statistical test used and presented the results appropriately, including confidence intervals or $p$-values $(k=47)$. However, only 14 studies provided a discussion of the size of the effect that was found.

\section{Measurements}

The majority of studies included in this systematic review measured loneliness using a version of the University of California Los Angeles (UCLA) Loneliness Scale $(k=29)$. A self-constructed ad hoc measure (including single-item measures) was used in $k=8$ studies. Five studies used a version of the De Jong Gierveld Loneliness Scale. Three studies used other loneliness measures (e.g., daily loneliness, expressions of loneliness on Twitter). Four studies measured objective social isolation instead of loneliness, asking the participants to report, for example, the number of days in self-isolation.

\section{Prevalence of Loneliness Before and During the COVID-19 Pandemic}

There was great heterogeneity in reporting prevalence rates of loneliness before and during the COVID-19 pandemic. Some studies reported percentages of people indicating that they did feel lonely versus did not feel lonely using a selfselected cut-off value (e.g., Stickley et al., 2021). Other studies reported percentages of people for different response categories of the loneliness scale (e.g., 7.22\% felt often lonely, and $23.63 \%$ felt sometimes lonely; Li \& Wang, $2020)$ or the mean and standard deviation $(S D)$ of loneliness scores before and during the pandemic and conducted a statistical test to compare them (e.g., Rogers et al., 2021). The conclusions of the different studies regarding significant changes in loneliness from before to during the pandemic were also heterogeneous. For example, Luchetti et al. (2020) reported no significant mean-level changes in loneliness across assessments. However, Ausín et al. (2021) found a significant mean-level increase of loneliness between the measures before and during the pandemic. Table 1 provides an overview of the main conclusion regarding changes in loneliness from before to during the pandemic. Most studies reported an increase in loneliness, which will be discussed in more detail later.

\section{Correlates of Loneliness During the COVID-19 Pandemic}

Numerous different correlates of loneliness during the pandemic have been investigated in the studies included in this systematic review. For a full overview, see Table S1. Across studies, the risk and protective factors that are known from before the pandemic remained risk or protective factors during the pandemic. For example, and similar to wellestablished findings (e.g., Algren et al., 2020; Buecker et al., 2021; Luo et al., 2012; Pinquart \& Sörensen, 2000), socioeconomic status was negatively associated with loneliness, and poor health or health issues was positively associated with loneliness (Bu et al., 2020a; Pan et al., 2021). Most correlates of loneliness reported in the included articles could be subsumed under "mental health variables," including stress, anxiety, depression, sleep problems, drug use, and general worries. These variables were consistently positively associated with loneliness during the pandemic, which matches previous studies from before the pandemic (e.g., Beutel et al., 2017; de Lange et al., 2020; Matthews et al., 2016). One included study explicitly compared correlates of loneliness before and during the pandemic (Bu et al., 2020a) and found that the risk factors for loneliness were nearly identical before and during the pandemic.

\section{Discussion}

The systematic review explored loneliness and its correlates during the COVID-19 pandemic. Out of 12 studies that reported prevalence rates of loneliness before and during the pandemic, nine found an increase in loneliness. Two found stable trends, and one found a decrease in loneliness. The heterogeneity of results could have resulted from different response formats, cut-off criteria for considering a person as lonely, differential time lags between the measurement occasions, international/cultural differences, or different scales.

\section{Heterogeneity in the Study Designs and Results}

A strength of this review is that it considers studies from different countries and with diverse samples (e.g., regarding age). However, the heterogeneity of study designs and time lags between the measurement occasions considerably complicates the comparability of results. Most studies used only one measurement occasion of loneliness or social isolation during the pandemic. Therefore, they only present a snapshot of loneliness and social isolation in an environment that changes from day to day. For an exception, Buecker et al. (2020) found that, on average, loneliness slightly increased during the first 2 weeks of the COVID19 pandemic but decreased thereafter. A recent study from 
Table 1. Changes in loneliness from before to during the COVID-19 pandemic

\begin{tabular}{|c|c|c|c|c|c|c|}
\hline Authors & Study type & $\begin{array}{l}\text { Loneliness } \\
\text { trend }\end{array}$ & $\begin{array}{l}\text { Pre-pandemic } \\
\text { assessment }\end{array}$ & $\begin{array}{l}\text { Peri-pandemic } \\
\text { assessment }\end{array}$ & Age group & Country \\
\hline Luchetti et al. (2020) & Longitudinal study (2 assessments) & $\leftrightarrow$ & $\begin{array}{l}\text { January/ } \\
\text { February } 2020\end{array}$ & March 2020 & Various & USA \\
\hline McGinty et al. (2020) & Pooled cross-sectional study & $\uparrow$ & 2018 & April 2020 & Various & USA \\
\hline Ruggieri et al. (2020) & Longitudinal study (3 assessments) & $\uparrow$ & March $2020^{1}$ & $\begin{array}{l}\text { March } 2020 \\
\text { and April } 2020\end{array}$ & Various & Italy \\
\hline Rogers et al. (2021) & Longitudinal study (2 assessments) & $\uparrow$ & October 2019 & April 2020 & Various & USA \\
\hline Bu et al. (2020a) & Pooled cross-sectional study & $\uparrow$ & June 2019 & March 2020 & Various & Great Britain \\
\hline Bu et al. (2020b) & Longitudinal study (3+ assessments) & $\leftrightarrow^{3}$ & March $2020^{2}$ & $\begin{array}{l}\text { March } 2020 \\
\text { - May } 2020\end{array}$ & Various & Great Britain \\
\hline Folk et al. (2020) & Longitudinal study (2 assessments) & $\downarrow$ & February 2020 & April 2020 & Young adults & Various \\
\hline Guntuku et al. (2020) & Pooled cross-sectional study & $\uparrow^{4}$ & April 2019 & April 2020 & N/A & Various \\
\hline Heidinger \& Richter (2020) & Longitudinal study (2 assessments) & $\uparrow$ & April/July 2019 & $\begin{array}{l}\text { April and } \\
\text { May } 2020\end{array}$ & Older adults & Austria \\
\hline Krendl \& Perry (2020) & Longitudinal study (2 assessments) & $\uparrow$ & Summer/fall 2019 & April/May 2020 & Older adults & USA \\
\hline Lee et al. (2020) & Longitudinal study (2 assessments) & $\uparrow$ & January 2020 & April/May 2020 & Young adults & USA \\
\hline Van Tilburg et al. (2020) & Longitudinal study (2 assessments) & $\uparrow$ & $\begin{array}{l}\text { October/ } \\
\text { November } 2019\end{array}$ & May 2020 & Older adults & Netherlands \\
\hline
\end{tabular}

Note. Loneliness trend: $\downarrow=$ decrease; $\uparrow=$ increase; $\leftrightarrow=$ stable trend. ${ }^{1}$ The assessment took place on March 7-9, on March 11 the first lockdown measures were implemented. ${ }^{2}$ The assessment took place on March 11, which was 2 days before the national lockdown. ${ }^{3}$ On average, the loneliness trend was characterized as stable. However, four classes of loneliness were found, with slightly increasing loneliness in the highest loneliness group, slightly decreasing loneliness in the lowest loneliness group, and relatively stable loneliness in the middle two groups. ${ }^{4}$ Frequeny of loneliness expressions on Twitter were studied.

Switzerland (Seifert \& Hassler, 2020) found that loneliness increased after the Swiss government recommended contact restrictions and slightly decreased after these restrictions were eased. If one assumes that loneliness changes during the COVID-19 pandemic are non-linear, studies examining changes in loneliness at different time points will necessarily come to different conclusions. Suppose loneliness is assessed years before the pandemic and again in the first few weeks during the pandemic, where loneliness increased (Buecker et al., 2020): In that case, a study may report an increase from before to during the pandemic. However, if a study examines loneliness a few months later when loneliness levels have decreased already, no overall increase might be observed.

Studies that found stable or decreasing loneliness trends used a pre-pandemic measurement occasion relatively close to the implementation of the first pandemic-related measures. In contrast, most other studies used a prepandemic measure of loneliness from about a year before the pandemic. This finding raises the question of whether an increase in loneliness, as reported in those studies with an initial assessment of loneliness years or months before the pandemic, can be attributed to the pandemic or the anti-pandemic related measures.

Moreover, some studies reported differences in the loneliness trajectories for different groups. Bu et al. (2020b) found that people with higher baseline loneliness levels that is, pre-pandemic - showed increases in loneliness over the first few weeks of the lockdown. In contrast, people with lower levels of baseline loneliness showed decreases in loneliness. People with mid-levels of baseline loneliness showed relatively constant loneliness levels during the lockdown. Buecker and colleagues (2020) found that loneliness increased more strongly over time for parents than for people without children. Additionally, they found that loneliness increased more strongly over time with increasing age.

\section{Further Open Research Questions}

Loneliness, earlier defined as a discrepancy between desired and actual social relationships, increases if social relationships suffer qualitatively or quantitatively. One of the main assumptions - or possibly the main assumption in the discussion of increasing loneliness - is that due to the virus containment measures, the quality, and quantity of desired social interactions will suffer, leading to increased loneliness levels. However, none of the reviewed studies has examined this assumption. By definition, loneliness should only increase if virus containment measures severely impacted the perceived quantity or quality of social relations. Based on the systematic review, it remains unclear whether changes in loneliness co-occurred with changes in the quality and/or the quantity of social relationships as no single study included in this systematic review examined those processes. In the second part of this study, we, therefore, test for changes in the perceived quality and quantity of social relationships changes over time, when virus containment measures became more restrictive. 
In the next part of this article, we provide an empirical investigation, using high-resolution data on the quantity and quality of social relationships to enrich the systematic review findings. With this empirical investigation, we aim to enhance the understanding of changes in loneliness during the COVID-19 pandemic. As loneliness is defined as the perceived discrepancy between the quality and quantity of social relations (Perlman \& Peplau, 1981), we focus on these two perceptions. We investigate how these perceptions change during a time in which different virus containment measures (e.g., contact restrictions) were first implemented and intensified.

\section{Research Objective 2: Empirical Investigation}

\section{Methods}

For the second research objective, we used the Bochum Berlin COVID-19 Study data, a large-scale $(N=4,823)$ daily diary study conducted between March 16, 2020, and April 12, 2020. ${ }^{1}$ Data from this study have been published elsewhere (Buecker et al., 2020; Horstmann et al., 2021), but data analyzed in the current study have not been analyzed or published before.

\section{Recruiting Procedure}

Data were collected online in Germany using formr.org (Arslan et al., 2020). A diverse sample was recruited via online sources (e.g., Twitter, Facebook, Xing), media reporting (e.g., radio, newspapers), and personal contacts. After registering for the study and providing informed consent, participants completed a baseline survey. Subsequently, brief daily diary surveys were administered between 5:00 p.m. and 1:00 a.m. on four consecutive days, followed by a three-day break and a weekly review survey. The next survey cycle started the day after the weekly survey, consisting of four daily surveys, a break, and a weekly review survey. For a detailed description of the study procedure, see Buecker and colleagues (2020).

\section{Sample}

In the present article, we used data from March 16, 2020 (the beginning of the Bochum Berlin COVID-19 Study and the first day of the nationwide pandemic-related measures in Germany) until April 12, 2020. We excluded participants if they did not complete any daily diary survey on the perceived quality of social relationships or the perceived quantity of social relationships. Moreover, we excluded daily surveys if they were filled out later than 01:00 a.m.

The total sample consisted of $N=4,823$ participants $\left(M_{\text {age }}=37.91, S D_{\text {age }}=14.42\right.$, range $18-88$ years $)$. Our sample was predominantly female (77.67\%). Participants completed $n=39,990$ daily surveys ( $M d n=1,435$ surveys per day in which at least one of the two focal items (see below) was answered, with $M d n=8$ surveys per participant in which at least one of the two focal items was answered).

\section{Measures}

In the present article, the following sociodemographic characteristics of the sample from the baseline survey were used: age, gender, employment status, relationship status, household type (living alone vs. living with others), preexisting health conditions concerning COVID-19. Table S2 (OSM 2) presents an overview of these characteristics and the distribution of responses.

The quality of social relationships compared to before the pandemic was repeatedly assessed in the daily surveys using a single item ("Compared to times before the coronavirus outbreak, the quality of my social relationships was worse today"; $M=3.78, S D=0.97$ ). Quantity of social relationships compared to before the pandemic was repeatedly assessed in the daily surveys using a single item ("Compared to times before the coronavirus outbreak, I have interacted less with other people today"; $M=2.75$, $S D=1.07)$. Participants responded to these items on a 5 -point rating scale ranging from $1=$ totally disagree to $5=$ totally agree.

\section{Statistical Analyses}

We used multilevel models to predict the perceived quality and quantity of social relationships over time. Perceived quality and quantity of social relationships (Level 1) were nested within persons (at Level 2). The time variable was coded as days since the beginning of the study, with 0 indicating the middle of the study period (i.e., the third Monday, March 30, 2020). Moreover, we included a dummy variable, indicating whether the daily survey was administered on a weekday (Monday to Friday; coded with 0 ) or a weekend (Saturday/Sunday; coded with 1). We refer to this variable as the day of the week (DOW) dummy. All analyses were performed in $\mathrm{R}$ version 4.0.3 ( $\mathrm{R}$ Core Team, 2020). We used the R-packages lme 4 1.1-25 (Bates et al., 2015) and lmerTest 3.1-3 (Kuznetsova et al., 2017). We $z$ standardized our dependent variables perceived quality and quantity of social relationships on their overall mean and $S D$. Whenever we extended a model with additional effects (e.g., covariates or random slopes), we tested the

\footnotetext{
The data collection of the Bochum Berlin COVID-19 Study is still ongoing. However, to be able to compare our results to the previous results by Buecker et al. (2020) on loneliness, we choose the same time interval as they did in the current analyses.
} 
Table 2. Fixed effect estimates from random slopes models for quality of social relationships and the quantity of social relationships

\begin{tabular}{|c|c|c|c|c|c|c|}
\hline \multirow[b]{2}{*}{ Variables } & \multicolumn{3}{|c|}{ Quality of social relationships } & \multicolumn{3}{|c|}{ Quantity of social relationships } \\
\hline & Estimate & SE & $p$ & Estimate & SE & $p$ \\
\hline Intercept & 0.054 & 0.012 & $<.001$ & 0.040 & 0.011 & $<.001$ \\
\hline Days & 0.045 & 0.005 & $<.001$ & $-0.042^{\#}$ & 0.006 & $<.001$ \\
\hline Days $^{2}$ & -0.035 & 0.004 & $<.001$ & & & \\
\hline DOW: weekday & $-0.088^{\#}$ & 0.009 & $<.001$ & $-0.092^{\#}$ & 0.010 & $<.001$ \\
\hline
\end{tabular}

Note. SE = Standard Error; $p$ = Significance Level; DOW = Day of the Week Dummy; The variable days indicates the date of the assessment and is centered such that zero represents the beginning of the third week (March 30,2020). Significant random effects are flagged with a hashtag (\#). Empty cells indicate that this coefficient was not estimated in the model.

model fit improvement using chi-square difference tests. We only continued extending the model if the extended model converged and the model fit improved significantly $(p<.01)$. All analyses were performed separately for the two dependent variables.

First, we estimated a random intercept model in which the dependent variable was predicted by the linear time term days and the DOW-dummy (see above for how these variables were defined). This model was then extended by a quadratic time term $\left(\right.$ days $\left.^{2}\right)$ and later by a cubic time term $\left(\right.$ days $\left.^{3}\right)$. We used the chi-square deviance test to test whether the more complex model fit the data better than the simpler model.

Next, we estimated random slopes for all included Level 1 predictors simultaneously (i.e., days, days ${ }^{2}$, and DOWdummy). If a model did not converge, we reduced the number of predictors for which we estimated random slopes, beginning with the higher-order terms (i.e., days ${ }^{3}$, days ${ }^{2}$ ), until the model converged. After establishing the Level 1 predictors and random slopes, we included all Level 2 covariates as additional predictors simultaneously in both models. In a final step, we added a cross-level interaction between days and one Level 2 predictor. This step was performed for all Level 2 predictors separately while controlling for all other Level 2 covariates.

\section{Results}

The quality of social relationships was predicted by days, days $^{2}$, and the DOW-dummy in our final best-fitting model. Random slopes were estimated for the DOW-dummy. Regarding the quantity of social relationships, the dependent variable was predicted by days and the DOW-dummy in our final best-fitting model. Random slopes were estimated for days, and the DOW-dummy (see Table 2).

The agreement to the item "Compared to times before the coronavirus outbreak, the quality of my social relationships was worse today." slightly increased over the 4 weeks of the study period. However, agreement stagnated, as the significant (negative) quadratic coefficient days $^{2}$ shows (see Figures 2A and $3 \mathrm{~A}$ ). The agreement to the item "Compared to times before the coronavirus outbreak, I have interacted less with other people today." linearly decreased over the 4 weeks of the study period (see Figures 2B and 3B).

To quantify changes in the perceived quality and quantity of social relationships over time, we estimated the change in $S D$ during Week 1 and 2 and Week 3 and 4 of the study. However, as the last day of the study was a Sunday, this would have led to a biased estimate due to the additional effect of the weekend. Therefore, we estimated the values for the last day as if it was a weekday. The perceived quality of social relationships increased during Week 1 and 2 by $S D=0.20$ and then decreased by $S D=-0.04$. Thus, the effect leveled off. The perceived quantity of social relationships decreased linearly by $S D=-0.08$ during Week 1 and 2 and again by $S D=-0.08$ during Week 3 and 4 , showing a negative linear trend.

We included cross-level interactions only for the model predicting the quantity of social relationships, as only this model contained random slopes for the day variable (Heisig \& Schaeffer, 2019). The cross-level interactions between days and age, gender, family status, and occupational status (Figure 4 and Table S3) were significant. The cross levelinteraction of household type and pre-existing health conditions concerning COVID-19 (Table S3; OSM 2) with days was not significant. The positive cross-level interaction between days and age indicates that with increasing age, the reduction of the quantity of social relationships compared to before the pandemic decreased less strongly over time (Figure 4A; Model 1 in Table S3). The cross-level interaction between days and gender indicates that the reduction of the quantity of social relationships compared to before the pandemic decreased more strongly over time for men than for women (Figure 4B; Model 2 in Table S3). There was no significant difference in the trajectories of women and people who identified as non-binary. The cross-level interaction between days and family status indicates that the reduction of the quantity of social relationships compared to before the pandemic decreased 
A

Quality of Social Relationships

Lowess and individual lines

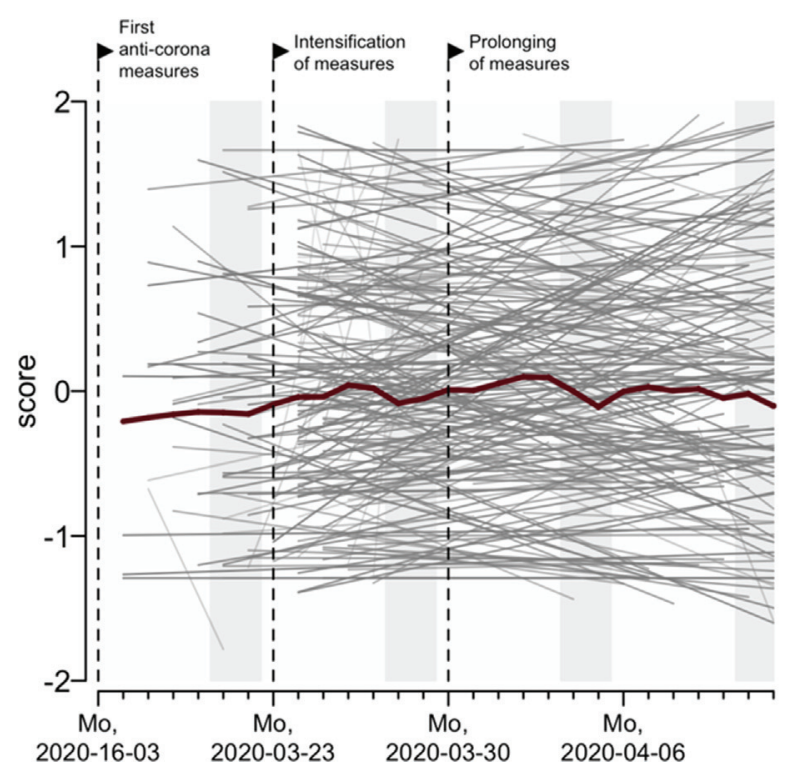

B

\section{Quantity of Social Relationships}

Lowess and individual lines

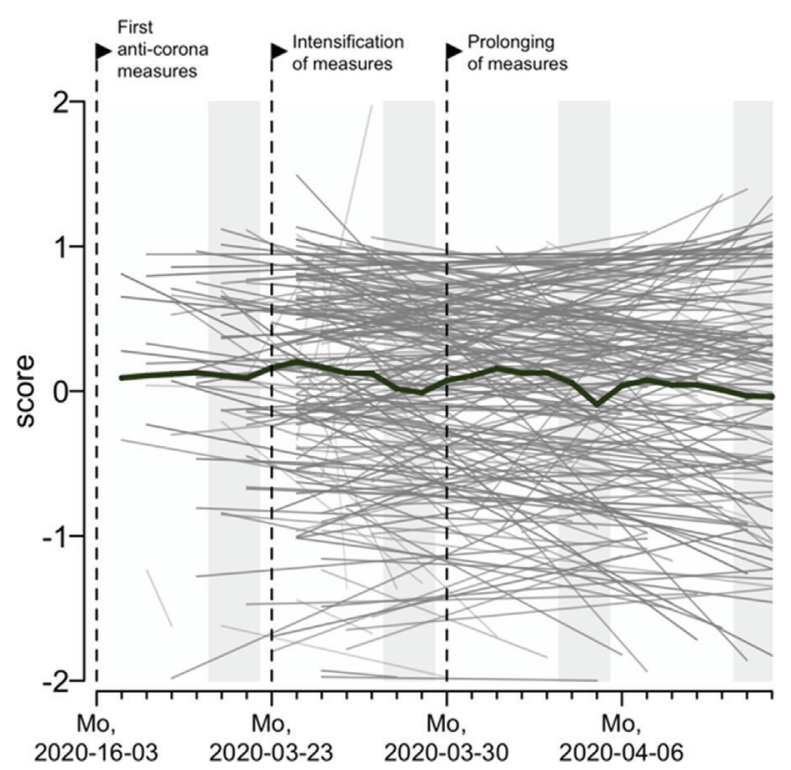

Figure 2. (A) Changes in daily-measured quality of social relationships (z-standardized) and (B) quantity of social relationships ( $z$-standardized) throughout the study. Gray lines represent individual predicted levels of the dependent variables (z-standardized) for 250 randomly selected participants. The bold lines represent LOWESS curves. Gray bars indicate weekends.

A

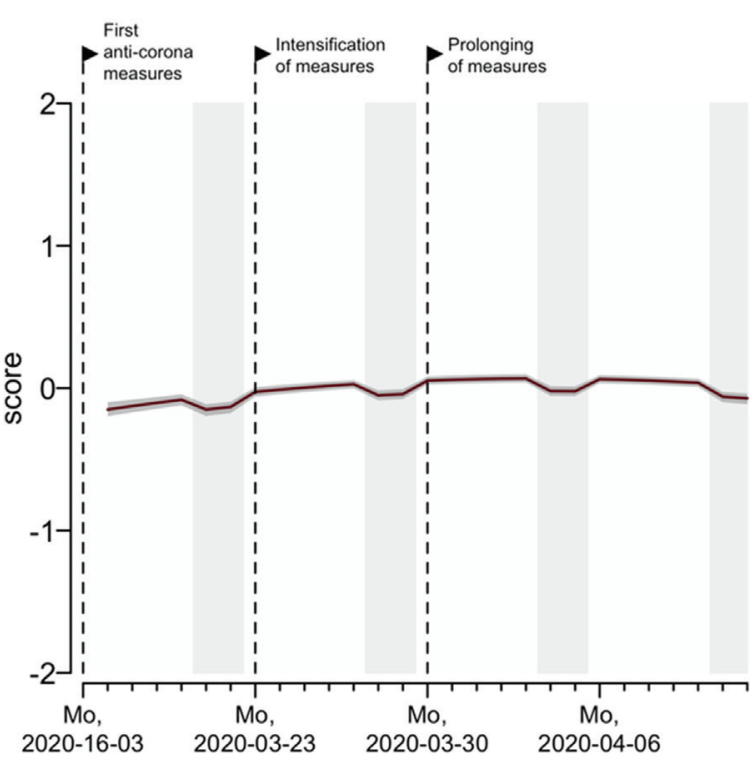

B

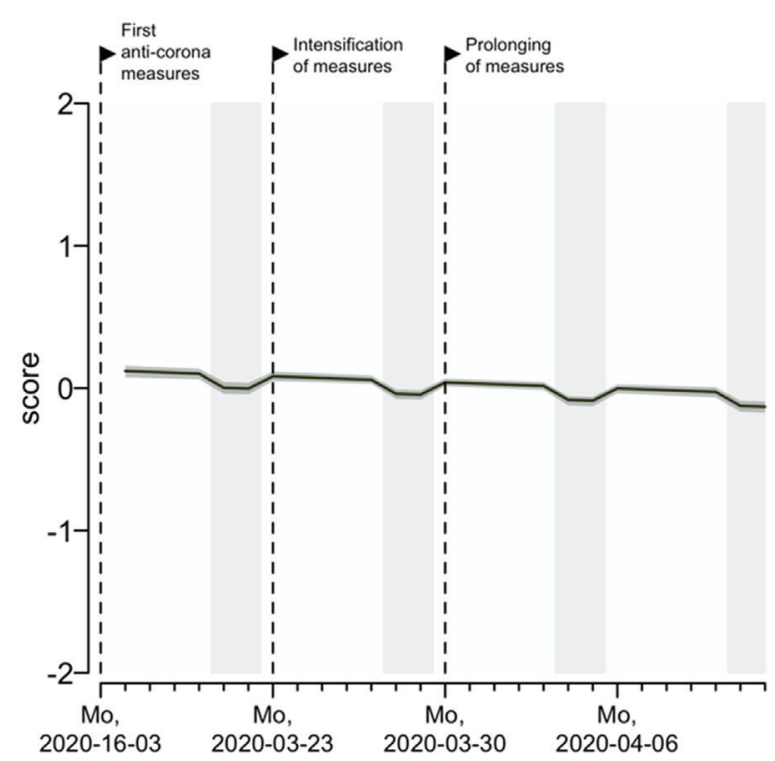

Figure 3. (A) Changes in daily-measured quality of social relationships (z-standardized) and (B) quantity of social relationships ( $z$-standardized) throughout the study. The bold line represents predicted values from the final random coefficient model, flanked by $99 \%$ confidence intervals (gray area). Gray bars indicate weekends.

more strongly for singles than for people who are in a romantic relationship (Figure 4C; Model 3 in Table S3). There was no significant difference in the trajectories of people in a romantic relationship and people who are divorced or widowed. Finally, the cross-level interaction between days and occupational status indicates that the 

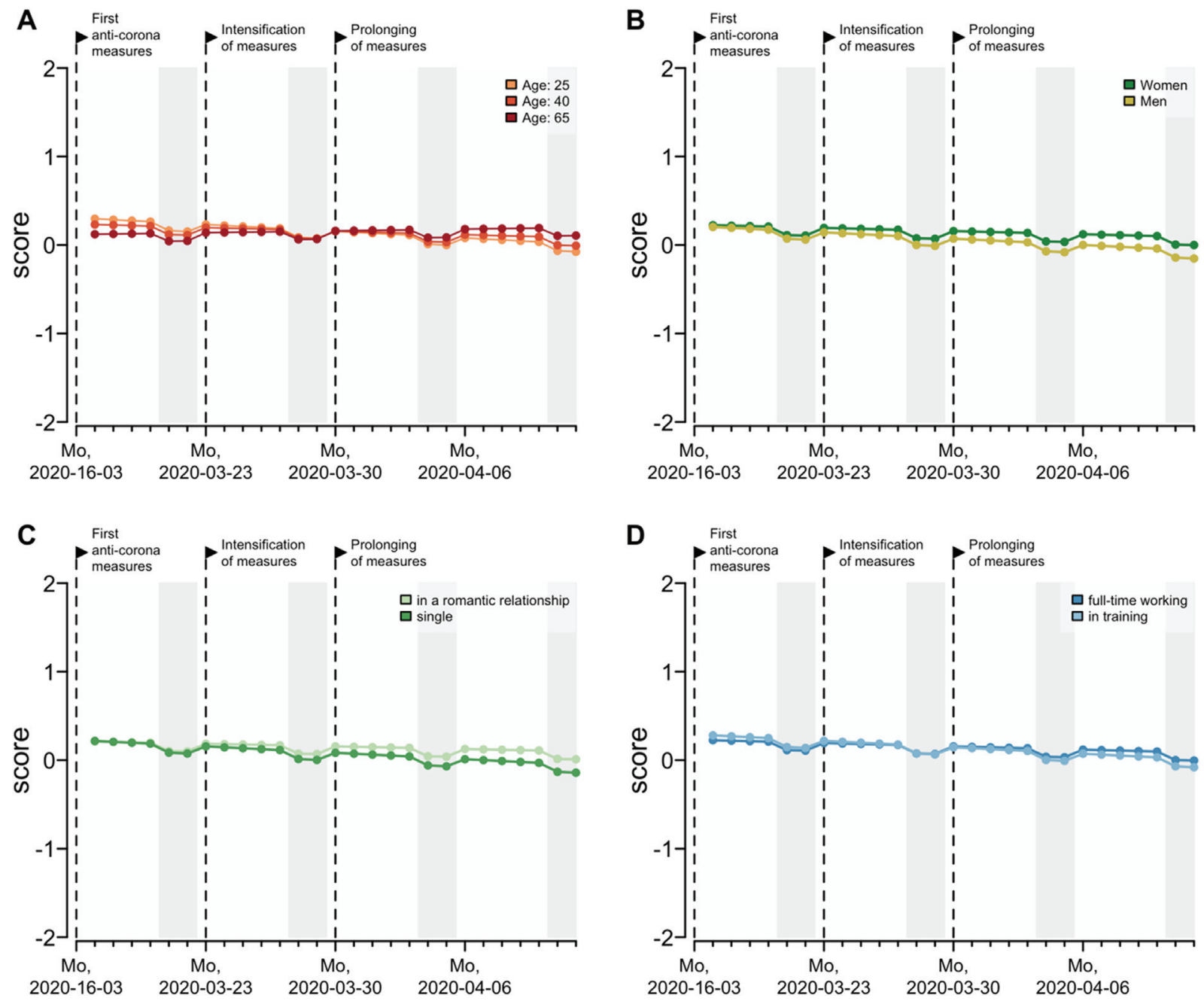

Figure 4. Only significant cross-level interactions are plotted. Predicted levels of the reduction of the quantity of social relationships compared to before the pandemic ( $z$-standardized) for: (A) different ages; (B) women and men; (C) singles and people in a romantic relationship; (D) people who work full-time and people who are in training. Gray bars indicate weekends. We controlled for the other investigated Level 2 covariates.

reduction of the quantity of social relationships compared to before the pandemic decreased more strongly for people in training than for people who were working full-time (Figure 4D; Model 4 in Table S3). There was no significant difference in the trajectories of people working full-time and people either not working, part-time working, or reporting another occupational status.

\section{Discussion}

Our empirical investigation showed that - at the beginning of the study (Mid-March 2020; the implementation of the first pandemic-related measures in Germany) - the quality of social relationships was on average perceived worse during the pandemic than before the pandemic. This perception got slightly stronger over the first 2 weeks of the study but stagnated thereafter. This stagnation is remarkable as the pandemic-related restrictions that were intensified over the first 2 weeks (Mid-March 2020 until End-March 2020) remained constant in Weeks 3 and 4. People may have perceived a "social shock" at the beginning of the pandemic but no longer felt their loss of quality in social relationships as strongly after a while. Besides, people may have found alternative (digital) ways of communication, reducing the actual loss of quality in social relationships.

Regarding the quantity of social relationships, participants reported on average that they had fewer social interactions at the beginning of the study compared to before the pandemic. This perceived reduction in the quantity of social interactions linearly decreased over time. People have reduced the quantity of their social contacts, especially in the beginning. However, with each additional week, the 
reported number of social interactions (including digital interactions) seems to have returned toward a normal, pre-pandemic level. A social psychological theory of loneliness describes loneliness as a social deficiency - a mismatch between one's desired and one's achieved a level of social contact (Peplau \& Perlman, 1979). This mismatch may have been exacerbated during the COVID-19 pandemic by contact restrictions. Future research may use the exploratory findings from this study on changes in the quality and quantity of social relationships to strengthen or update this theory on loneliness.

\section{General Discussion}

The systematic review explored loneliness and social isolation during the COVID-19 pandemic. Most studies included in this systematic review were cross-sectional and thus do not allow a conclusion regarding changes from before to during the COVID-19 pandemic. The few longitudinal studies mainly reported increases in loneliness, especially when the pre-pandemic measurement occasions were months or years before the COVID-19 pandemic. Studies with pre-pandemic measures weeks or days before the COVID-19 pandemic reported rather stable or even decreasing loneliness trends.

The primary study quality assessment revealed a lack of studies following nationally representative or well-stratified samples from before to during the pandemic. Moreover, studies examining changes in loneliness and social isolation from before to during the pandemic rarely reported and discussed the effect sizes of such changes. Although the narrative of increases in loneliness and social isolation during the pandemic may sound compelling, transparent communication of effect sizes is important to avoid exaggerated claims if effect sizes are small. This is particularly important when media and politicians pick up the results of these empirical studies.

The review also revealed that longitudinal studies with multiple assessments of loneliness and social isolation during the COVID-19 pandemic are rare. If one wants to understand the consequences of the virus containment measures, high-resolution studies with multiple measurement occasions during and after the pandemic are needed. This is especially important to assess the mid-term and long-term psychological changes in response to the pandemic. As loneliness is related to negative physical and mental health (e.g., Beutel et al., 2017; Holt-Lunstad et al., 2015), potentially critical developments in these outcomes need further monitoring.

Our current empirical investigation showed that, on average, the quality of social relationships was perceived worse than before the pandemic. This perception got slightly stronger over the first 2 weeks of the COVID-19 pandemic but stagnated thereafter, indicating habituation to the new circumstances. Regarding the quantity of social relationships, participants reported on average that they had fewer social interactions at the beginning of the study compared to before the pandemic. This perceived reduction in the quantity of social interactions linearly decreased over time, indicating that a progression of the number of social interactions toward the baseline level happened.

\section{Correlates of Loneliness and Changes in the Quality and Quantity of Social Relationships}

In the systematic review, we identified several correlates of loneliness. The broader picture of loneliness correlates during the pandemic mirrors the picture already known from before the pandemic. It is noteworthy that many studies examined mental health outcomes (e.g., anxiety, depression, insomnia) associated with loneliness during the COVID-19 pandemic. This selection of variables illustrates the scientific and public concern that mental health may be at risk during the COVID-19 pandemic (e.g., Xiong et al., 2020).

In the empirical investigation, we identified four predictors of changes in the perceived reduction of the quantity of social relationships. With increasing age, the perceived reduction of the quantity of social relationships compared to before the pandemic decreased less strongly over time. Previous research showed that older people and people who worried more about a COVID-19 infection were more likely to voluntarily reduce interaction with family members, friends, and colleagues or classmates than younger people and people who worried less about an infection (Chou et al., 2020). However, it is also conceivable that younger and middle-aged people inevitably have more social interactions due to their professional activities than older people who are already retired.

The perceived reduction of the quantity of social relationships compared to before the pandemic decreased more strongly for men than for women. Previous research found that women were more likely to take health precautions, including social distancing and staying at home, than men (Clark et al., 2020). Our results suggest that the perceived loss of social interaction quantity decreased more strongly for men than for women over the study period. This effect could be attributed both to a different perception of social relationships between men and women (e.g., women register more quickly when their social relationships change) or to different social behavior (e.g., the pandemic-related measures impacted the everyday life of women more than that of men). 
The perceived reduction of the quantity of social relationships compared to before the pandemic decreased more strongly for singles than for people who are in a romantic relationship. Previous research showed that using dating applications for chatting and setting up virtual dates increased in the early phase of the COVID-19 pandemic compared to 2019 (Coombe et al., 2020). Singles may have begun to seek out (digital) social contacts earlier because social contact reduction was less bearable for them.

The perceived reduction of the quantity of social relationships compared to before the pandemic decreased more strongly for people in training (i.e., university students or people in vocational training) than for people working full-time. Previous research found a rather complex pattern of changes in university students' social network structures during the COVID-19 pandemic. While face-to-face interactions decreased, digital communication was widespread (Elmer et al., 2020). Moreover, Elmer and colleagues found that students reported relatively stable friendship networks from before the pandemic to during the pandemic and increased informational and emotional support. Thus, people in training seemed to be able to compensate for a potential loss in the quantity of social contacts more easily using digital contact, likely because they were used to this kind of contact (i.e., higher digital literacy). However, the abovestated explanations for differences in the trajectories of social contact quantity are mostly speculative, and further research is needed to better understand these findings.

\section{Limitations}

The systematic review has some limitations. First, the description of the primary study findings was narrative. A statistical aggregation of the primary study findings was not feasible due to the high heterogeneity across studies in measuring loneliness and social isolation. Second, all studies included employed online surveys. This procedure is, of course, reasonable as the aim was to reduce social contact with other people (e.g., an interviewer) during the COVID19 pandemic. However, people with poor internet accessibility or poor technological skills were likely underrepresented in the included studies. Moreover, people from low-income countries were underrepresented in the studies. Another concern was the majority of women in most studies. Thus, the studied samples are most likely not representative of the general population, limiting the generalizability of the summarized findings. Third, our search strategy was restricted to studies published in the English or German language, which may involve a risk for bias. Fourth, this systematic review covered studies that were published until November 2020. However, the COVID-19 pandemic and its related measures may have long-term consequences for loneliness, which could not be covered in the present systematic review. Finally, the majority of the reviewed studies were from North America or Europe. However, virus containment measures might differ in their impact on the perceived quality and quantity of social interactions depending on the culture. Future research may explore these cultural effects and use more ethnically diverse samples.

Our empirical examination of changes in the quality and quantity of social relationships has several strengths, such as daily measures, a large sample, and the ideal time focus from the beginning of the first pandemic-related measures. Still, there are some important limitations. We used convenient sampling and collected the data online, which produces problems regarding the generalizability of the results to the general public and may involve a risk for bias (e.g., opt-in bias; Pierce et al., 2020).

\section{Conclusion}

We provide the first systematic review on changes in the prevalence of loneliness and social isolation and their correlates during the COVID-19 pandemic. Moreover, we examined day-to-day changes in the quality and quantity of social relationships during the COVID-19 pandemic using a large-scale daily diary study from Germany. In the systematic review, we identified that most studies reported a higher prevalence of loneliness during the pandemic than before the pandemic. However, studies using multiple assessments during the pandemic also provide evidence for recovery from an initial increase in loneliness at the beginning of the pandemic. This "recovery process" was found in the empirical investigation of the daily diary study on changes in the quality and quantity of social relationships: the perceived loss in relationship quality from the beginning of the COVID-19 pandemic stagnated, and the number of social interactions seemed to have increased over time. However, these data captured only short-term changes in social relationships. It is important to vigilantly observe how the quality and quantity of social relationships and ultimately loneliness evolve during and after the COVID-19 pandemic.

\section{References}

Algren, M. H., Ekholm, O., Nielsen, L., Ersbøll, A. K., Bak, C. K., \& Andersen, P. T. (2020). Social isolation, loneliness, socioeconomic status, and health-risk behaviour in deprived neighbourhoods in Denmark: A cross-sectional study. SSM - Population Health, 10, Article 100546. https://doi.org/10.1016/j.ssmph. 2020.100546

Arslan, R. C., Walther, M. P., \& Tata, C. S. (2020). formr: A study framework allowing for automated feedback generation and complex longitudinal experience-sampling studies using $\mathrm{R}$. Behavior Research Methods, 52(1), 376-387. https://doi.org/ 10.3758/s13428-019-01236-y 
Ausín, B., González-Sanguino, C., Castellanos, M. Á., \& Muñoz, M. (2021). Gender-related differences in the psychological impact of confinement as a consequence of COVID-19 in Spain. Journal of Gender Studies, 30(1), 29-38. https://doi.org/10.1080/ 09589236.2020 .1799768

Bates, D., Maechler, M., Bolker, B., \& Walker, S. (2015). Fitting linear mixed-effects models using Ime4. Journal of Statistical Software, 67(1), 1-48. https://doi.org/10.18637/jss.v067.i01

Beutel, M. E., Klein, E. M., Brähler, E., Reiner, I., Jünger, C., Michal, M., Wiltink, J., Wild, P. S., Münzel, T., Lackner, K. J., \& Tibubos, A. N. (2017). Loneliness in the general population: Prevalence, determinants and relations to mental health. BMC Psychiatry, 17(1), 1-7. https://doi.org/10.1186/s12888-017-1262-x

Bu, F., Steptoe, A., \& Fancourt, D. (2020a). Who is lonely in lockdown? Cross-cohort analyses of predictors of loneliness before and during the COVID-19 pandemic. Public Health, 186, 31-34. https://doi.org/10.1016/j.puhe.2020.06.036

Bu, F., Steptoe, A., \& Fancourt, D. (2020b). Loneliness during a strict lockdown: Trajectories and predictors during the COVID19 pandemic in 38,217 United Kingdom adults. Social Science \& Medicine, 265, Article 113521. https://doi.org/10.1016/ j.socscimed.2020.113521

Buecker, S., Ebert, T., Götz, F. M., Entringer, T. M., \& Luhmann, M. (2021). In a lonely place: Investigating regional differences in loneliness. Social Psychological and Personality Science, 12(2), 147-155. https://doi.org/10.1177/1948550620912881

Buecker, S., Horstmann, K. T., Krasko, J., Kritzler, S., Terwiel, S., Kaiser, T., \& Luhmann, M. (2020). Changes in daily loneliness for German residents during the first four weeks of the COVID19 pandemic. Social Science \& Medicine, 265, Article 113541. https://doi.org/10.1016/j.socscimed.2020.113541

Chou, W.-P., Wang, P.-W., Chen, S.-L., Chang, Y.-P., Wu, C.-F., Lu, W.-H., \& Yen, C.-F. (2020). Voluntary reduction of social interaction during the COVID-19 pandemic in Taiwan: Related factors and association with perceived social support. International Journal of Environmental Research and Public Health, 17(21), Article 8039. https://doi.org/10.3390/ijerph17218039

Clark, C., Davila, A., Regis, M., \& Kraus, S. (2020). Predictors of COVID-19 voluntary compliance behaviors: An international investigation. Global Transitions, 2, 76-82. https://doi.org/ 10.1016/j.glt.2020.06.003

Coombe, J., Yuh Shiong Kong, F., Bittleston, H., Williams, H., Tomnay, J., Vaisey, A., Malta, S., Goller, J. L., Temple-Smith, M., Bourchier, L., Lau, A., Chow, E. P. F., \& Hocking, J. S. (2020). Love during lockdown: Findings from an online survey examining the impact of COVID-19 on the sexual health of people living in Australia. Sexually Transmitted Infections, 1-7. https:// doi.org/10.1136/sextrans-2020-054688

De Lange, A.-M. G., Kaufmann, T., Quintana, D. S., Winterton, A., Westlye, L. T., \& Ebmeier, K. P. (2020). Risk factors associated with loneliness, social isolation, and neuroticism in the UK Biobank cohort. PsyArXiv. https://doi.org/10.31234/osf.io/q8kjv

Elmer, T., Mepham, K., \& Stadtfeld, C. (2020). Students under lockdown: Comparisons of students' social networks and mental health before and during the COVID-19 crisis in Switzerland. PLoS One, 15(7), Article e0236337. https://doi. org/10.1371/journal.pone.0236337

Folk, D., Okabe-Miyamoto, K., Dunn, E., \& Lyubomirsky, S. (2020). Did social connection decline during the first wave of COVID-19? The role of extraversion. Collabra: Psychology, 6(1), Article 37. https://doi.org/10.1525/collabra.365

Guntuku, S. C., Sherman, G., Stokes, D. C., Agarwal, A. K., Seltzer, E., Merchant, R. M., \& Ungar, L. H. (2020). Tracking mental health and symptom mentions on Twitter during COVID-19. Journal of General Internal Medicine, 35(9), 27982800. https://doi.org/10.1007/s11606-020-05988-8
Heidinger, T., \& Richter, L. (2020). The effect of COVID-19 on loneliness in the Elderly. An empirical comparison of pre-and peri-pandemic loneliness in community-dwelling Elderly. Frontiers in Psychology, 11, Article 585308. https://doi.org/10.3389/ fpsyg. 2020.585308

Heisig, J. P., \& Schaeffer, M. (2019). Why you should always include a random sope for the lower-level variable involved in a cross-level interaction. European Sociological Review, 35(2), 258-279. https://doi.org/10.1093/esr/jcy053

Holt-Lunstad, J., Smith, T. B., Baker, M., Harris, T., \& Stephenson, D. (2015). Loneliness and social isolation as risk factors for mortality: A meta-analytic review. Perspectives on Psychological Science, 10(2), 227-237. https://doi.org/10.1177/ 1745691614568352

Horstmann, K. T., Buecker, S., Krasko, J., Kritzler, S., \& Terwiel, S. (2021). Who does or does not use the "Corona-Warn-App" and why? European Journal of Public Health, 31(1), 49-51. https:// doi.org/10.1093/eurpub/ckaa239

Klein, E. (2020, March 12). Coronavirus will also cause a loneliness epidemic. Vox. https://www.vox.com/2020/3/12/21173938/ coronavirus-covid-19-social-distancing-elderly-epidemic-isolationquarantine

Krendl, A. C., \& Perry, B. L. (2020). The impact of sheltering in place during the COVID-19 pandemic on older adults' social and mental well-being. The Journals of Gerontology: Series B, Article gbaa110. https://doi.org/10.1093/geronb/gbaa110

Kuznetsova, A., Brockhoff, P. B., \& Christensen, R. H. B. (2017). ImerTest package: Tests in linear mixed effects models. Journal of Statistical Software, 82(13), 1-26. https://doi.org/10.18637/ jss.v082.i13

Lee, C. M., Cadigan, J. M., \& Rhew, I. C. (2020). Increases in loneliness among young adults during the COVID-19 pandemic and association with increases in mental health problems. Journal of Adolescent Health, 67(5), 714-717. https://doi.org/ 10.1016/j.jadohealth.2020.08.009

Li, L. Z., \& Wang, S. (2020). Prevalence and predictors of general psychiatric disorders and loneliness during COVID-19 in the United Kingdom. Psychiatry Research, 291, Article 113267. https://doi.org/10.1016/j.psychres.2020.113267

Luchetti, M., Lee, J. H., Aschwanden, D., Sesker, A., Strickhouser, J. E., Terracciano, A., \& Sutin, A. R. (2020). The trajectory of loneliness in response to COVID-19. American Psychologist, 75(7), 897-908. https://doi.org/10.1037/amp0000690

Luo, Y., Hawkley, L. C., Waite, L., \& Cacioppo, J. T. (2012). Loneliness, health, and morality in old age: A national longitudinal study. Social Science Medicine, 74(6), 907-914. https:// doi.org/10.1016/j.socscimed.2011.11.028.Loneliness

Matthews, T., Danese, A., Wertz, J., Odgers, C. L., Ambler, A., Moffitt, T. E., \& Arseneault, L. (2016). Social isolation, loneliness and depression in young adulthood: A behavioural genetic analysis. Social Psychiatry and Psychiatric Epidemiology, 51(3), 339-348. https://doi.org/10.1007/s00127-016-1178-7

McGinty, E. E., Presskreischer, R., Han, H., \& Barry, C. L. (2020). Psychological distress and loneliness reported by US adults in 2018 and April 2020. Journal of the American Medical Association, 324(1), 93-94. https://doi.org/10.1001/jama.2020.9740

Moher, D., Liberati, A., Tetzlaff, J., Altman, D. G., \& Group, The. P. R. I. S. M. A. (2009). Preferred reporting items for systematic reviews and meta-analyses: The PRISMA statement. PLOS Medicine, 6(7), Article e1000097. https://doi.org/10.1371/journal. pmed.1000097

Pan, Y., Yang, Z., Han, X., \& Qi, S. (2021). Family functioning and mental health among secondary vocational students during the COVID-19 epidemic: A moderated mediation model. Personality and Individual Differences, 171, Article 110490. https://doi.org/ 10.1016/j.paid.2020.110490 
Peplau, L. A., \& Perlman, D. (1979). Blueprint for a social psychological theory of loneliness. In M. Cook \& G. Wilson (Eds.), Love and attraction (pp. 99-108). Pergamon Press.

Perlman, D., \& Peplau, L. A. (1981). Toward a social psychology of loneliness. In R. Gilmour \& S. Duck (Eds.), Personal relationships: 3 Relationships in disorder (pp. 31-56). Academic Press.

Pierce, M., McManus, S., Jessop, C., John, A., Hotopf, M., Ford, T., Hatch, S., Wessely, S., \& Abel, K. M. (2020). Says who? The significance of sampling in mental health surveys during COVID-19. The Lancet Psychiatry, 7(7), 567-568. https://doi. org/10.1016/S2215-0366(20)30237-6

Pinquart, M., \& Sörensen, S. (2000). Influences of socioeconomic status, social network, and competence on subjective wellbeing in later life: A meta-analysis. Psychology and Aging, 15(2), 187-224. https://doi.org/10.1037/0882-7974.15.2.187

R Core Team. (2020). R: A language and environment for statistical computing. R Foundation for Statistical Computing. https:// www.r-project.org/

Rogers, A. A., Ha, T., \& Ockey, S. (2021). Adolescents' perceived socio-emotional impact of COVID-19 and implications for mental health: Results from a US-based mixed-methods study. Journal of Adolescent Health, 68(1), 43-52. https://doi.org/ 10.1016/j.jadohealth.2020.09.039

Ruggieri, S., Ingoglia, S., Bonfanti, R. C., \& Lo Coco, G. (2020). The role of online social comparison as a protective factor for psychological wellbeing: A longitudinal study during the COVID-19 quarantine. Personality and Individual Differences, Article 110486. https://doi.org/10.1016/j.paid.2020.110486

Seifert, A., \& Hassler, B. (2020). Impact of the COVID-19 pandemic on loneliness among older adults. Frontiers in Sociology, 5, Article 590935. https://doi.org/10.3389/fsoc.2020.590935

Stefana, A., Youngstrom, E. A., Hopwood, C. J., \& Dakanalis, A. (2020). The COVIDL19 pandemic brings a second wave of social isolation and disrupted services. European Archives of Psychiatry and Clinical Neuroscience, 270(6), 785-786. https://doi. org/10.1007/s00406-020-01137-8

Stickley, A., Matsubayashi, T., \& Ueda, M. (2021). Loneliness and COVID-19 preventive behaviours among Japanese adults. Journal of Public Health, 43(1), 53-60. https://doi.org/ 10.1093/pubmed/fdaa151

Van Tilburg, T. G., Steinmetz, S., Stolte, E., van der Roest, H., \& de Vries, D. H. (2020). Loneliness and mental health during the COVID-19 pandemic: A study among Dutch older adults. The Journals of Gerontology: Series B, Article gbaa111. https://doi. org/10.1093/geronb/gbaa111

Wells, G. A., Shea, B., O'Connell, D., Peterson, J., Welch, V., Losos, M., \& Tugwell, P. (2004). The Newcastle-Ottawa Scale (NOS) for assessing the quality of nonrandomised studies in meta-analyses. http://www.ohri.ca/programs/clinical_epidemiology/oxford.asp

WHO. (2020). Pandemie der Coronavirus-Krankheit (COVID-19) [Coronavirus disease pandemic (COVID-19)]. https://www. euro.who.int/de/health-topics/health-emergencies/coronavirus-covid-19/novel-coronavirus-2019-ncov

Xiong, J., Lipsitz, O., Nasri, F., Lui, L. M. W., Gill, H., Phan, L., ChenLi, D., lacobucci, M., Ho, R., Majeed, A., \& Mclntyre, R. S. (2020). Impact of COVID-19 pandemic on mental health in the general population: A systematic review. Journal of Affective Disorders, 277, 55-64. https://doi.org/10.1016/j.jad.2020.08.001

\section{Acknowledgments}

We express our great appreciation to Aline Beckers and Chiara Förster for their assistance with this article.

\section{Conflict of Interest}

The authors declare no conflict of interest.

\section{Authorship}

Susanne Buecker conceptualized the study, wrote the manuscript, performed the coding of the data, collected the empirical data, conducted the analyses. Kai T. Horstmann gave feedback to the manuscript, performed the coding of the data, collected the empirical data, conducted the analyses.

\section{Open Data}

We summarize the study characteristics and main study findings of all included articles in Table S1 at the Open Science Framework (OSF; https://osf.io/w25nk/?view_only=0434f9ce80bb46b08e529 8e362a481f0).

\section{Funding}

The authors did not receive specific funding for this work. Open access publication enabled by Ruhr University Bochum.

\section{ORCID}

Susanne Buecker

iDhttps://orcid.org/0000-0003-3443-5400

\section{Susanne Buecker}

Department of Psychology

Ruhr University Bochum

Universitätsstraße 150

44801 Bochum

Germany

susanne.buecker@rub.de

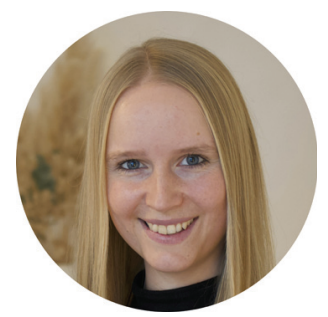

Susanne Buecker is a postdoctoral researcher at the Department of Psychology at Ruhr University Bochum, Germany. Her research focuses on loneliness, well-being, and personality traits. She is particularly interested in changes in these constructs in everyday life and across the life span. Further research interests include meta-analytic techniques.

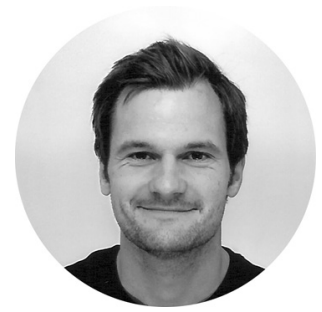

Kai T. Horstmann is a junior professor at Humboldt-Universität zu Berlin, Germany. He obtained his PhD in 2018 on the interplay of personality, affect, situations, and behavior. He currently investigates the description, measurement, and prediction of daily behaviors, cognitions, and feelings using daily diary and experience sampling methods.

\section{History}

Received December 24, 2020

Revision received April 6, 2021

Accepted May 11, 2021

Published online January 5, 2022 